\title{
Low-density expansion for the two-dimensional electron gas
}

\author{
Francesca Sauli and Peter Kopietz \\ Institut für Theoretische Physik, Universität Frankfurt, \\ Max-von-Laue Strasse 1, 60438 Frankfurt, Germany
}

(Dated: August 23, 2006)

\begin{abstract}
We show that in two dimensions $(2 D)$ a systematic expansion of the self-energy and the effective interaction of the dilute electron gas in powers of the two-body $T$-matrix $T_{0}$ can be generated from the exact hierarchy of functional renormalization group equations for the one-particle irreducible vertices using the chemical potential as flow parameter. Due to the interference of particle-particle and particle-hole channels at order $T_{0}^{2}$, in $2 D$ the ladder approximation for the self-energy is not reliable beyond the leading order in $T_{0}$. We also discuss two-body scattering in vacuum in arbitrary dimensions from the renormalization group point of view and argue that the singular interaction proposed by Anderson [Phys. Rev. Lett. 65, 2306 (1990)] cannot be ruled out on the basis of the ladder approximation.
\end{abstract}

PACS numbers: 71.10.Ca, 71.10.Hf, 73.43.Nq

Calculating the physical properties of strongly interacting electrons in two spatial dimensions $(2 D)$ remains one of the big challenges of condensed matter theory. Most authors agree that the normal state of interacting electrons in $2 D$ is a Fermi liquid, at least for weak interactions. There are even rigorous proofs that at weak coupling certain two-dimensional models for interacting electrons (including the repulsive Hubbard model away from half filling) are Fermi liquids above an energy scale which is non-perturbative in the coupling constant 1]. However, due to a lack of controlled methods, one cannot exclude the possibility that for sufficiently strong interactions Fermi liquid theory breaks down in $2 D$. Anderson has argued repeatedly [2, 3] that due to non-perturbative effects which are neglected by the usual field-theoretical machinery of many-body theory, the normal state of the two-dimensional Hubbard model is not a Fermi liquid for arbitrary strength of the interaction. This scenario has been criticized early on by Engelbrecht and Randeria [4], who pointed out that a calculation of the selfenergy $\Sigma(\boldsymbol{k}, \omega)$ within the ladder approximation (LAP) predicts Fermi liquid behavior. However, the LAP has some rather peculiar and most likely unphysical features; in particular, the limiting behavior of $\Sigma(\boldsymbol{k}, \omega)$ for small frequencies $\omega$ and for wave-vectors $\boldsymbol{k}$ in the vicinity of the Fermi surface $\boldsymbol{k}_{F}$ is very sensitive to the order in which the limits $\omega \rightarrow 0$ and $\boldsymbol{k} \rightarrow \boldsymbol{k}_{F}$ are taken [5, 6].

Unfortunately, going beyond the LAP is very difficult, because the particle-hole scattering channels have to be taken into account on the same footing with the particleparticle scattering channel retained within the LAP, i.e., one has to solve coupled Bethe-Salpeter equations in several channels. In this work we shall take a fresh look at this problem using a formally exact hierarchy for renormalization group (RG) flow equations for the one-particle irreducible vertices [7].
Functional $R G$ in the spin-singlet channel. Starting point of our analysis are the formally exact RG flow equations for the irreducible self-energy $\Sigma_{\Lambda}(K)$ and the antisymmetrized effective interaction vertex $\Gamma_{\Lambda}^{(4)}\left(K_{1}^{\prime} \sigma_{1}^{\prime}, K_{2}^{\prime} \sigma_{2}^{\prime} ; K_{2} \sigma_{2}, K_{1} \sigma_{1}\right)$. Here $K=(\boldsymbol{k}, i \omega)$ denotes wave-vector and Matsubara frequency, and $\sigma_{i}$ are the spin projections. $\Lambda$ is a cutoff scale separating the low-energy from the high-energy degrees of freedom, such that for $\Lambda=\Lambda_{0}$ the low-energy fluctuations are suppressed. For the Hubbard model on a $D$-dimensional hyper-cubic lattice with lattice spacing $a$ and on-site repulsion $U$ the bare interaction is $\Gamma_{\Lambda_{0}}^{(4)}=\left(\delta_{\sigma_{1}, \sigma_{1}^{\prime}} \delta_{\sigma_{2}, \sigma_{2}^{\prime}}-\right.$ $\left.\delta_{\sigma_{1}, \sigma_{2}^{\prime}} \delta_{\sigma_{2}, \sigma_{1}^{\prime}}\right) \tilde{U}_{0}$, where $\tilde{U}_{0}=a^{D} U$. As we iterate the RG, the flowing effective interaction develops a spin-triplet component, so that in general

$$
\begin{aligned}
& \Gamma_{\Lambda}^{(4)}\left(K_{1}^{\prime} \sigma_{1}^{\prime}, K_{2}^{\prime} \sigma_{2}^{\prime} ; K_{2} \sigma_{2}, K_{1} \sigma_{1}\right) \\
& =\left(\delta_{\sigma_{1}, \sigma_{1}^{\prime}} \delta_{\sigma_{2}, \sigma_{2}^{\prime}}-\delta_{\sigma_{1}, \sigma_{2}^{\prime}} \delta_{\sigma_{2}, \sigma_{1}^{\prime}}\right) \Gamma_{\Lambda}^{s}\left(K_{1}^{\prime}, K_{2}^{\prime} ; K_{2}, K_{1}\right) \\
& +\left(\delta_{\sigma_{1}, \sigma_{1}^{\prime}} \delta_{\sigma_{2}, \sigma_{2}^{\prime}}+\delta_{\sigma_{1}, \sigma_{2}^{\prime}} \delta_{\sigma_{2}, \sigma_{1}^{\prime}}\right) \Gamma_{\Lambda}^{t}\left(K_{1}^{\prime}, K_{2}^{\prime} ; K_{2}, K_{1}\right)
\end{aligned}
$$

The singlet part $\Gamma_{\Lambda}^{s}\left(K_{1}^{\prime}, K_{2}^{\prime} ; K_{2}, K_{1}\right)$ is symmetric under the exchange $K_{1}^{\prime} \leftrightarrow K_{2}^{\prime}$ or $K_{1} \leftrightarrow K_{2}$, while the triplet part $\Gamma_{\Lambda}^{t}\left(K_{1}^{\prime}, K_{2}^{\prime} ; K_{2}, K_{1}\right)$ is antisymmetric and therefore vanishes for $K_{1}^{\prime}=K_{2}^{\prime}$ or $K_{1}=K_{2}$. The RG flow equation for $\Gamma_{\Lambda}^{(4)}$ contains also mixed terms $\Gamma_{\Lambda}^{t} \Gamma_{\Lambda}^{s}$ and a term involving the six-point vertex $\Gamma_{\Lambda}^{(6)}$. However, $\Gamma_{\Lambda}^{t}$ and $\Gamma_{\Lambda}^{(6)}$ are both irrelevant in the RG sense [7], so that we shall neglect them. Within this approximation, the $\mathrm{RG}$ flow of the self-energy is determined by

$$
\partial_{\Lambda} \Sigma_{\Lambda}(K)=\int_{K^{\prime}} \dot{G}_{\Lambda}\left(K^{\prime}\right) \Gamma_{\Lambda}^{s}\left(K, K^{\prime} ; K^{\prime}, K\right)
$$

and the effective interaction satisfies at zero temperature 


$$
\begin{aligned}
\partial_{\Lambda} \Gamma_{\Lambda}^{s}\left(K_{1}^{\prime}, K_{2}^{\prime} ; K_{2}, K_{1}\right)=- & \int_{K}\left[\dot{G}_{\Lambda}(K) G_{\Lambda}\left(K_{1}+K_{2}-K\right)+G_{\Lambda}(K) \dot{G}_{\Lambda}\left(K_{1}+K_{2}-K\right)\right] \\
& \times \Gamma_{\Lambda}^{s}\left(K_{1}^{\prime}, K_{2}^{\prime} ; K_{1}+K_{2}-K, K\right) \Gamma_{\Lambda}^{s}\left(K, K_{1}+K_{2}-K ; K_{2}, K_{1}\right) \\
- & \frac{1}{2} \int_{K}\left\{\left[\dot{G}_{\Lambda}(K) G_{\Lambda}\left(K+K_{1}-K_{1}^{\prime}\right)+G_{\Lambda}(K) \dot{G}_{\Lambda}\left(K+K_{1}-K_{1}^{\prime}\right)\right]\right. \\
& \left.\times \Gamma_{\Lambda}^{s}\left(K_{1}^{\prime}, K+K_{1}-K_{1}^{\prime} ; K, K_{1}\right) \Gamma_{\Lambda}^{s}\left(K_{2}^{\prime}, K ; K+K_{1}-K_{1}^{\prime}, K_{2}\right)+\left(K_{1}^{\prime} \leftrightarrow K_{2}^{\prime}\right)\right\},
\end{aligned}
$$

where $\int_{K}=\int \frac{d^{D} k}{(2 \pi)^{D}} \frac{d \omega}{2 \pi}$, and we have introduced the cutoff-dependent propagator

$$
G_{\Lambda}(K)=\Theta_{\Lambda}(\boldsymbol{k})\left[i \omega-\xi_{\boldsymbol{k}}-\Sigma_{\Lambda}(K)\right]^{-1},
$$

and the single scale propagator

$$
\dot{G}_{\Lambda}(K)=\left[1+G_{\Lambda}^{0}(K) \Sigma_{\Lambda}(K)\right]^{-2} \partial_{\Lambda} G_{\Lambda}^{0}(K) .
$$

Here $\Theta_{\Lambda}(\boldsymbol{k})$ is some cutoff function which suppresses the low-energy modes, normalized such that $\Theta_{0}(\boldsymbol{k})=1$ and $\Theta_{\infty}(\boldsymbol{k})=0$. The cutoff-dependent non-interacting propagator is $G_{\Lambda}^{0}(K)=\Theta_{\Lambda}(\boldsymbol{k})\left[i \omega-\xi_{\boldsymbol{k}}\right]^{-1}$, with $\xi_{\boldsymbol{k}}=\epsilon_{\boldsymbol{k}}-\mu$, where $\epsilon_{\boldsymbol{k}}$ is the bare energy dispersion and $\mu$ is the chemical potential. The first term on the right-hand side of Eq. (3) is the contribution from the particle-particle channel, while the other two terms correspond to the particle-hole channels (also called zero-sound channels).

Ladder approximation. In the limit of vanishing density only the particle-particle channel contributes to the effective interaction. At low densities, it is then reasonable to calculate the self-energy in LAP, where the contribution of the particle-hole channels to the effective interaction in Eq. (3) is simply ignored. It is then advantageous to introduce total and relative energy-momenta as independent variables, $P=K_{1}+K_{2}=K_{1}^{\prime}+K_{2}^{\prime}$, $Q=\left(K_{1}-K_{2}\right) / 2, Q^{\prime}=\left(K_{1}^{\prime}-K_{2}^{\prime}\right) / 2$, and define

$$
\Gamma_{\Lambda}^{s}\left(\frac{P}{2}+Q^{\prime}, \frac{P}{2}-Q^{\prime} ; \frac{P}{2}-Q, \frac{P}{2}+Q\right)=\Gamma_{\Lambda}\left(Q^{\prime}, Q ; P\right) .
$$

With this approximation Eq. (3) reduces to

$$
\begin{aligned}
\partial_{\Lambda} \Gamma_{\Lambda}\left(Q^{\prime}, Q ; P\right)= & -2 \int_{K} \dot{G}_{\Lambda}\left(\frac{P}{2}+K\right) G_{\Lambda}\left(\frac{P}{2}-K\right) \\
& \times \Gamma_{\Lambda}\left(Q^{\prime}, K ; P\right) \Gamma_{\Lambda}(K, Q ; P) .
\end{aligned}
$$

Assuming that the bare interaction is independent of the frequency-part of $Q$ and $Q^{\prime}$, this remains also true for the renormalized interaction, so that we may write $\Gamma_{\Lambda}\left(Q^{\prime}, Q ; P\right)=\Gamma_{\Lambda}\left(\boldsymbol{q}^{\prime}, \boldsymbol{q} ; P\right)$. If we replace the flowing Green functions in Eq. (7) by the non-interacting ones, the frequency sum is easily carried out,

$$
\begin{aligned}
& \partial_{\Lambda} \Gamma_{\Lambda}\left(\boldsymbol{q}^{\prime}, \boldsymbol{q} ; P\right)=\int \frac{d^{D} k}{(2 \pi)^{D}} \partial_{\Lambda}\left[\Theta_{\Lambda}\left(\frac{\boldsymbol{p}}{2}+\boldsymbol{k}\right) \Theta_{\Lambda}\left(\frac{\boldsymbol{p}}{2}-\boldsymbol{k}\right)\right] \\
& \times \frac{1-f\left(\xi_{\frac{p}{2}+\boldsymbol{k}}\right)-f\left(\xi_{\frac{p}{2}-\boldsymbol{k}}\right)}{i \bar{\omega}-\xi_{\frac{p}{2}+\boldsymbol{k}}-\xi_{\frac{p}{2}-\boldsymbol{k}}} \Gamma_{\Lambda}\left(\boldsymbol{q}^{\prime}, \boldsymbol{k} ; P\right) \Gamma_{\Lambda}(\boldsymbol{k}, \boldsymbol{q} ; P),
\end{aligned}
$$

where $P=(\boldsymbol{p}, i \bar{\omega})$, and $f(\xi)=\Theta(-\xi)$ is the Fermi function at zero temperature. Eq. (8) is equivalent with the Bethe-Salpeter equation for the effective interaction in the particle-particle channel [8]. We recover the LAP if we assume that $\Gamma_{\Lambda}\left(\boldsymbol{q}^{\prime}, \boldsymbol{q} ; P\right)=\Gamma_{\Lambda}(P)$ is independent of $\boldsymbol{q}$ and $\boldsymbol{q}^{\prime}$, in which case Eq. (8) becomes an ordinary differential equation, $\partial_{\Lambda} \Gamma_{\Lambda}(P)=\dot{\chi}_{\Lambda}(P) \Gamma_{\Lambda}^{2}(P)$, where

$$
\begin{aligned}
\dot{\chi}_{\Lambda}(P)= & \int \frac{d^{D} k}{(2 \pi)^{D}} \partial_{\Lambda}\left[\Theta_{\Lambda}\left(\frac{\boldsymbol{p}}{2}+\boldsymbol{k}\right) \Theta_{\Lambda}\left(\frac{\boldsymbol{p}}{2}-\boldsymbol{k}\right)\right] \\
& \times \frac{1-f\left(\xi_{\frac{p}{2}+\boldsymbol{k}}\right)-f\left(\xi_{\frac{p}{2}-\boldsymbol{k}}\right)}{i \bar{\omega}-\xi_{\frac{p}{2}+\boldsymbol{k}}-\xi_{\frac{p}{2}-\boldsymbol{k}}} .
\end{aligned}
$$

The solution of the above differential equation with initial condition $\Gamma_{\Lambda_{0}}(P)=\tilde{U}_{0}$ yields the usual LAP for the effective interaction,

$$
\Gamma(P)=\Gamma_{\Lambda=0}(P)=\tilde{U}_{0}\left[1+\tilde{U}_{0} \chi(P)\right]^{-1},
$$

where the pair susceptibility $\chi(P)=\int_{0}^{\Lambda_{0}} d \Lambda \dot{\chi}_{\Lambda}(P)$ is given by

$$
\begin{aligned}
& \chi(P)=-\int \frac{d^{D} k}{(2 \pi)^{D}} {\left[1-\Theta_{\Lambda_{0}}\left(\frac{\boldsymbol{p}}{2}+\boldsymbol{k}\right) \Theta_{\Lambda_{0}}\left(\frac{\boldsymbol{p}}{2}-\boldsymbol{k}\right)\right] } \\
& \times \frac{1-f\left(\xi_{\frac{p}{2}+\boldsymbol{k}}\right)-f\left(\xi_{\frac{p}{2}-\boldsymbol{k}}\right)}{i \bar{\omega}-\xi_{\frac{p}{2}+\boldsymbol{k}}-\xi_{\frac{p}{2}-\boldsymbol{k}}} .
\end{aligned}
$$

Zero density limit. In this limit $\mu \rightarrow 0$ and we may approximate $\epsilon_{\boldsymbol{k}}=\boldsymbol{k}^{2} /(2 m)$, where $m$ is some effective band mass. The Fermi functions $f\left(\xi_{\frac{p}{2} \pm \boldsymbol{k}}\right)$ then vanish. Using for simplicity a sharp cutoff in momentum space, $\Theta_{\Lambda}(\boldsymbol{k})=\Theta(|\boldsymbol{k}|-\Lambda)$, we find from Eq. (8) that the twobody $T$-matrix $T_{\Lambda}\left(\boldsymbol{q}^{\prime}, \boldsymbol{q} ; i \bar{\omega}\right) \equiv \Gamma_{\Lambda}\left(\boldsymbol{q}^{\prime}, \boldsymbol{q} ; \boldsymbol{p}=0, i \bar{\omega}\right)$ in vacuum satisfies the flow equation

$$
\begin{aligned}
\partial_{\Lambda} T_{\Lambda}\left(\boldsymbol{q}^{\prime}, \boldsymbol{q}, i \bar{\omega}\right)= & -\int \frac{d^{D} k}{(2 \pi)^{D}} \frac{\delta(|\boldsymbol{k}|-\Lambda)}{i \bar{\omega}-\Lambda^{2} / m} \\
& \times T_{\Lambda}\left(\boldsymbol{q}^{\prime}, \boldsymbol{k}, i \bar{\omega}\right) T_{\Lambda}(\boldsymbol{k}, \boldsymbol{q}, i \bar{\omega}) .
\end{aligned}
$$

The initial condition is $T_{\Lambda_{0}}\left(\boldsymbol{q}^{\prime}, \boldsymbol{q}, i \bar{\omega}\right)=\tilde{U}_{\boldsymbol{q}^{\prime}-\boldsymbol{q}}$, where $\tilde{U}_{\boldsymbol{k}}$ is the Fourier transform of the bare interaction. Eq. (12) is the RG version of the Lippmann-Schwinger equation for the $T$-matrix of elementary scattering theory [9]. The low-energy behavior of the $T$-matrix defines the $s$-wave scattering length $a_{s}$ via $T(0,0, i 0)=$ 


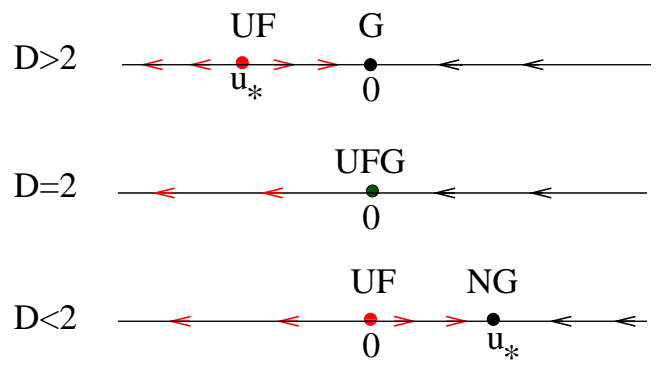

FIG. 1: (Color online) RG flow of the rescaled zero energy part $u_{l}=m K_{D} \Lambda^{D-2} T_{\Lambda}(0,0, i 0)$ of the two-body $T$-matrix.

$\gamma_{D} a_{s}^{D-2} / m$, where $\gamma_{D}$ is a numerical constant $\left(\gamma_{3}=\right.$ $4 \pi)$. Introducing the dimensionless coupling constant $u_{l}=m K_{D} \Lambda^{D-2} T_{\Lambda}(0,0, i 0)=K_{D} \gamma_{D}\left(a_{s} \Lambda\right)^{D-2}$, where $l=\ln \left(\Lambda_{0} / \Lambda\right)$ and $K_{D}=2^{1-D} \pi^{-D / 2} / \Gamma(D / 2)$ is the surface area of the $D$-dimensional unit sphere divided by $(2 \pi)^{D}$, we obtain from Eq. (12) the RG flow equation

$$
\partial_{l} u_{l}=\epsilon u_{l}-u_{l}^{2}, \epsilon=2-D .
$$

The corresponding flow of $u_{l}$ is shown in Fig. 1. For $D>2$ the RG flow has a stable Gaussian fixed point $\mathrm{G}$ at vanishing interaction, and an unstable fixed point UF at finite negative interaction $u_{*}=\epsilon<0$. At this point the zero energy $T$-matrix diverges for $\Lambda \rightarrow 0$, $T_{\Lambda}(0,0, i 0) \propto u_{*} \Lambda^{2-D} \rightarrow-\infty$, so that UF corresponds to the unitary Fermi gas in the limit of vanishing density [10]. For $D=2$ both fixed points $U F$ and $G$ merge into a single point UFG at zero interaction. Finally, for $D<2$ the unitary fixed point UF has zero interaction, because in this case an infinitesimal attractive interaction leads to a zero energy bound state 11]. However, there is a new non-Gaussian fixed point NG for finite repulsive interaction $u_{*}=\epsilon>0$, which resembles the Wilson-Fisher fixed point below four dimensions [12. We now show that this fixed point is characterized by a finite phase shift $\delta_{0}(0)=-\pi \epsilon / 2$ for $s$-wave scattering at zero energy. Setting again $\tilde{U}_{\boldsymbol{k}}=\tilde{U}_{0}$, the solution of Eq. (12) is

$$
T_{0}(i \bar{\omega})=T_{\Lambda=0}\left(\boldsymbol{q}^{\prime}, \boldsymbol{q}, i \bar{\omega}\right)=\tilde{U}_{0}\left[1+\tilde{U}_{0} \chi_{0}(i \bar{\omega})\right]^{-1},
$$

where from Eq. (11) we obtain

$$
\chi_{0}(i \bar{\omega})=-\int \frac{d^{D} k}{(2 \pi)^{D}} \frac{\Theta\left(\Lambda_{0}-|\boldsymbol{k}|\right)}{i \bar{\omega}-\boldsymbol{k}^{2} / m} .
$$

For $D<2$ the integral is convergent for $\Lambda_{0} \rightarrow \infty$, and we obtain for real frequencies,

$$
\begin{aligned}
\chi_{0}(\omega+i 0)= & \pi \nu_{D}\left(|\omega|, m_{r}\right)\left\{\Theta(-\omega)[\sin (\pi \epsilon / 2)]^{-1}\right. \\
& +\Theta(\omega)[\cot (\pi \epsilon / 2)+i]\}
\end{aligned}
$$

where $\nu_{D}\left(\omega, m_{r}\right)=K_{D} m_{r}\left(2 m_{r} \omega\right)^{-\epsilon / 2}$ is the density of states of a free particle with reduced mass $m_{r}=m / 2$. In $2 D$, where the density of states $\nu_{2}(m)=m /(2 \pi)$ is constant, we have

$$
\chi_{0}(\omega+i 0)=\pi \nu_{2}\left(m_{r}\right)\left[\ln \left(E_{0} /|\omega|\right)+i \Theta(\omega)\right],
$$

where $E_{0}=\Lambda_{0}^{2} / m$. The phase shift for $s$-wave scattering in the spin-singlet channel, defined via [4, 13] $T_{0}(\omega+i 0)=$ $-\left|T_{0}(\omega+i 0)\right| e^{i \delta_{0}(\omega)}$, is for $\omega>0$ given by

$$
\delta_{0}(\omega)=-\arctan \{\pi g(\omega) /[1+\pi g(\omega) \cot (\pi \epsilon / 2)]\}
$$

where $g(\omega)=\nu_{D}\left(\omega, m_{r}\right) \tilde{U}_{0}$ is the relevant dimensionless coupling. Because $g(0)=\infty$ for $D<2$ due to the divergence of the density of states, we conclude that $\delta_{0}(0)=-\pi \epsilon / 2$ for any finite $\tilde{U}_{0}>0$.

Low-density expansion in $2 D$. If we approximate the effective interaction in Eq. (2) by $\Gamma(P)$ given in Eq. (10) and ignore self-energy corrections to the propagators, we obtain the usual LAP for the self-energy [4, 5]. However, the LAP is not a systematic low-density expansion. In $2 D$ the relevant dimensionless low-density parameter is $\alpha=$ $\left[\ln \left(\Lambda_{0} / k_{F}\right)\right]^{-1}$. One should therefore expand the LAP in powers of $\alpha$. The self-energy within LAP up to order $\alpha^{2}$ has been evaluated by Bloom [14] using the pseudopotential method pioneered by Galitskii [8, 15]. In this method one first derives a non-linear integral equation relating the effective interaction in LAP to the on-shell two-body $T$-matrix in vacuum. Iteration of this integral equation generates the desired expansion in powers of $\alpha$.

The RG equations (2) and (3) offer an alternative way to generate the low-density expansion in $2 D$. The advantage of the $R G$ approach is that the contribution from the particle-hole channels neglected in LAP can be systematically included. The crucial point is that Eqs. (2) and (3) remain formally valid if we take the derivative with respect to any parameter appearing only in the free propagator [7]. In particular, we may choose $\Lambda=\mu$, in which case Eq. (2) and (3) describe the evolution of the self-energy and the effective interaction as we change the chemical potential. The propagator in the flow equations should then be replaced by $G_{\mu}(K)=\left[i \omega-\epsilon_{\boldsymbol{k}}+\mu-\Sigma_{\mu}(K)\right]^{-1}$, and the single scale propagator by $\dot{G}_{\mu}(K)=-G_{\mu}^{2}(K)$. The flow equation for the self-energy now reads

$$
\partial_{\mu} \Sigma_{\mu}(K)=\int_{P} \dot{G}_{\mu}(P-K) \Gamma_{\mu}\left(\frac{P}{2}-K, \frac{P}{2}-K ; P\right),
$$

and the flow equation for the effective interaction can be obtained by making analogous substitutions in Eq. (3). Assuming that an expansion in powers of $\mu$ is possible, we may generate this expansion by successive differentiation of Eq. (19) and the analogue of Eq. (3). For $\tilde{U}_{\boldsymbol{k}}=\tilde{U}_{0}$ the initial conditions at $\mu=0$ are $\Sigma_{\mu=0}(K)=0$ and $\Gamma_{\mu=0}\left(\frac{P}{2}-K, \frac{P}{2}-K ; P\right)=T_{0}\left(i \bar{\omega}-\frac{p^{2}}{4 m}\right) \equiv T(P)$. The leading term in the expansion of the self-energy is

$$
\Sigma_{\mu}(K)=\mu \int_{P} \dot{G}_{0}(P-K) T(P)+O\left(\mu^{2}\right),
$$

and from Eqs. (3) and (6) we obtain for the effective 
interaction

$$
\begin{aligned}
& \Gamma_{\mu}\left(Q^{\prime}, Q ; P\right)=T(P)-\mu T^{2}(P)\left[\partial_{\mu} \chi(P)\right]_{\mu=0} \\
& -\frac{\mu}{2} \int_{K}\left\{\left[\dot{G}_{0}(K) G_{0}\left(K+Q+Q^{\prime}\right)\right.\right. \\
& \left.\quad+G_{0}(K) \dot{G}_{0}\left(K+Q+Q^{\prime}\right)\right] T\left(K+Q+\frac{P}{2}\right) \\
& \left.\quad \times T\left(K+Q^{\prime}+\frac{P}{2}\right)+\left(Q^{\prime} \rightarrow-Q^{\prime}\right)\right\}+O\left(\mu^{2}\right),
\end{aligned}
$$

where the pair susceptibility $\chi(P)$ at finite $\mu$ is given in Eq. (11). The second term on the right-hand side of Eq. (21) is due to the $\mu$-dependence of the effective interaction in LAP, while the last term is the contribution from the particle-hole channels neglected in LAP.

Due to the non-analytic $\mu$-dependence of the density of states for $D \neq 2$ the above expansion in powers of $\mu$ is only possible in $D=2$. To see this more clearly, let us explicitly evaluate the first term on the right-hand side of Eq. (20). The result can be written as

$$
\Sigma_{\mu}(\boldsymbol{k}, i \omega) \approx \rho_{0}(\mu) T_{0}\left(i \omega-\frac{\boldsymbol{k}^{2}}{4 m}\right),
$$

where $\rho_{0}(\mu)=\mu \nu_{2}(m)$. In fact, Eq. (22) is the leading term in the expansion in powers of $T_{0}$ for arbitrary $D$ if we identify $\rho_{0}(\mu) \equiv(2 / D) \mu \nu_{D}(\mu, m) \propto \mu^{D / 2}$ with the density (per spin projection) in the absence of interactions. The non-analyticity in $\mu$ for $D \neq 2$ is obvious.

If we approximate $T_{0}$ by the bare interaction $\tilde{U}_{0}$, then Eq. (22) reduces to the Fock correction to the selfenergy. In contrast to the usual LAP [4, 5], the particleparticle susceptibility is evaluated at vanishing density in Eq. (22). It is instructive to explicitly calculate the corresponding single-particle Green function $G(\boldsymbol{k}, i \omega)$ for $D \leq 2$. The relation between $\mu$ and Fermi momentum $k_{F}$ is then $\frac{k_{F}^{2}}{2 m}+\rho_{0}(\mu) T_{0}\left(-\frac{k_{F}^{2}}{4 m}\right)=\mu$. Expanding $G^{-1}(\boldsymbol{k}, i \omega)$ for small $\omega$ and $|\boldsymbol{k}|-k_{F}$ we obtain the Fermi liquid form

$$
G(\boldsymbol{k}, i \omega) \approx Z\left[i \omega-\left(k_{F} / m_{*}\right)\left(|\boldsymbol{k}|-k_{F}\right)\right]^{-1},
$$

with quasi-particle residue $Z=1-\epsilon^{2} g^{2}[\epsilon+g]^{-2}$ and effective mass renormalization $m / m_{*}=1-\frac{1}{2} \epsilon^{2} g^{2}[\epsilon+g]^{-2}$. Here $g=\nu_{D}\left(\mu, m_{r}\right) \tilde{U}_{0}$, and in $2 D$ we should replace $\epsilon \rightarrow \alpha=\left[\ln \left(\Lambda_{0} / k_{F}\right)\right]^{-1}$. For $g \rightarrow \infty$ we obtain $Z \approx 1-\epsilon^{2}$ and $m / m_{*}=1-\epsilon^{2} / 2$. This agrees qualitatively with the results by Bloom [14], who obtained different numerical coefficients on front of the correction terms, which is due to the fact that his approximation amounts to retaining also the second term on the right-hand side of Eq. (21). However, to order $T_{0}^{2}$ the particle-hole contributions to Eq. (21) compete with the particle-particle channel retained in Ref. [14], so that the approximation employed by Bloom is inconsistent. In this point we agree with Anderson [3], but for different reasons: there is no mathematical mistake in Bloom's analysis, but in $2 D$ the LAP is inconsistent beyond the leading order in $T_{0}$.
In summary, we have reconsidered the low-density expansion for the electron gas in dimensions $D \leq 2$ using functional RG methods. At vanishing density, we have rewritten the Lippmann-Schwinger equation for the two-body $T$-matrix in vacuum as a $\mathrm{RG}$ flow equation and have pointed out that for $D<2$ this equation has a non-Gaussian fixed point characterized by a finite $s$ wave phase shift. At low densities in $2 D$ a systematic expansion of the self-energy and of the effective interaction in powers of the two-body $T$-matrix can be generated with the help of the functional RG flow equations for the irreducible vertices using the chemical potential as flow parameter. We have argued that in $2 D$ the LAP is inconsistent beyond the leading order in this expansion because already at order $T_{0}^{2}$ the contribution from the particle-hole channels competes with the particle-particle channel and gives rise to a complicated momentum- and frequency dependence of the effective interaction which still has to be explored. Because the LAP is not reliable in $2 D$, one cannot use the LAP to rule out that the true effective interaction in $2 D$ exhibits a singular dependence on the relative momenta, as proposed by Anderson [2, 3].

We thank M. Salmhofer for his comments on rigorous results for two-dimensional Fermi systems.

[1] M. Disertori and V. Rivasseau, Phys. Rev. Lett. 85, 361 (2000); W. Pedra and M. Salmhofer, in Proceedings of the 14th International Congress of Mathematical Physics, (Lisbon, 2003); G. Benfatto, A. Giuliani, and V. Mastropietro, cond-mat/0507686.

[2] P. W. Anderson, Phys. Rev. Lett. 64, 1839 (1990); ibid. 65, 2306 (1990); ibid. 66, 3226 (1991); ibid. 71, 1220 (1993).

[3] P. W. Anderson, cond-mat/0101417

[4] J. R. Engelbrecht and M. Randeria, Phys. Rev. Lett. 65, 1032 (1990); ibid. 66, 3226 (1991).

[5] H. Fukuyama and Y. Hasegawa, Prog. Theor. Phys. Suppl. 101, 441 (1990); H. Fukuyama, Y. Hasegawa, and O. Narikito, J. Phys. Soc. Jpn. 60, 2013 (1991).

[6] C. Halboth and W. Metzner, Phys. Rev. B 57, 8873 (1998).

[7] P. Kopietz and T. Busche, Phys. Rev. B 64, 155101 (2001); M. Salmhofer and C. Honerkamp, Prog. Theor. Phys. 105, 1 (2001).

[8] A. L. Fetter and J. D. Walecka, Quantum Theory of Many-Particle Systems, (McGraw-Hill, New York, 1971).

[9] See, for example, C. J. Pethick and H. Smith, BoseEinstein Condensation in Dilute Gases, (Cambridge University Press, Cambridge, 2002), chapter 5.

[10] T. Ho, Phys. Rev. Lett. 92, 090402 (2004).

[11] Y. Nishida and D. T. Son, Phys. Rev. Lett. 97, 050403 (2006); cond-mat/0607835.

[12] K. G. Wilson and M. E. Fisher, Phys. Rev. Lett. 28, 240 (1972).

[13] P. Nozières and S. Schmitt-Rink, J. Low Temp. Phys. 59, 195 (1985).

[14] P. Bloom, Phys. Rev. B 12, 125 (1975).

[15] V. M. Galitskii, Zh. Eksp. Teor. Fiz. 34, 151 (1958) [Sov. Phys. JETP 7, 104 (1958)]. 